\title{
Re-framing HCI through Local and Indigenous Perspectives
}

\author{
Jose Abdelnour-Nocera ${ }^{1}$, Masaaki Kurosu², Torkil Clemmensen ${ }^{3}$, Nic Bidwell ${ }^{4}$, \\ Ravi Vatrapu ${ }^{3}$, Heike Winschiers-Theophilus ${ }^{5}$, Vanessa Evers ${ }^{6}$, \\ Rüdiger Heimgärtner ${ }^{7}$, and Alvin $\mathrm{Yeo}^{8}$ \\ ${ }^{1}$ University of West London \\ ${ }^{2}$ The Open University of Japan \\ ${ }^{3}$ Copenhagen Business School \\ ${ }^{4}$ CSIR-Meraka \& Nelson Mandela Metropolitan University \\ ${ }^{5}$ Polytechnic of Namibia \\ ${ }^{6}$ University of Amsterdam \\ ${ }^{7}$ Intercultural User Interface Consulting Germany \\ ${ }^{8}$ Universiti Malaysia Sarawak \\ Jose. Abdelnour-Nocera@uwl.ac.uk \\ masaakikurosu@spa.nifty.com, tc.inf@cbs.dk, \\ nic.bidwell@gmail.com, rv.caict@cbs.dk, \\ heikew@polytechnic.edu.na, evers@uva.nl, \\ ruediger.heimgaertner@iuic.de, awyeo@acm.org
}

\begin{abstract}
This one-day workshop aims to present different local and indigenous perspectives from all over the world in order to lead into an international dialogue on re-framing concepts and models in HCI/Interaction Design. The target audience is HCI researchers and practitioners who have experience with working with culture and HCI. The expected outcome of the workshop is a) network building among the participants, b) a shortlist of papers that can be basis for a proposal for a special issue of the UAIS journal, and c) identify opportunities to develop a funded network or research proposal.
\end{abstract}

Keywords: Indigenous HCI, HCI theory and methodology, localization, globalization, cultural usability.

\section{Objective and Theme}

One of the current challenges for HCI as a discipline is addressing the tensions created between local cultures and the assumptions, priorities and values embedded in the HCI as a discipline. The objective of this workshop is to further our understanding of these issues and lead to practical recommendations for people researching and implementing HCI from an explicit indigenous perspective. Translating local knowledge into valid and useful HCI tools is not a simple problem, but one that requires re-defining and renegotiating disciplinary boundaries (and connections) and the subject and object of the interaction design. Focusing on local or indigenous awareness and practices in design pushes the envelope in a very exciting way. For instance, the democratic values of equal participation driving user-centred design are not necessarily shared by local communities which prioritize respecting the views of their leaders. Addressing these 